\title{
PIEMONTESISCH IM AOSTATAL ${ }^{1}$
}

Das Piemontesische zählt neben dem Frankoprovenzalishcen, dem Französischen, dem Italienischen und dem Walserdeutschen zu den heute noch im Aostatal vertreten Sprachen. Der Beitrag dokumentiert einerseits die sprachgescichtliche Bedeutung des Piemontesischen seit etwa 1850 und skizziert andererseits seinen heutigen Status und seine innerhalb der valdostanischen Sprachlandschaft verbliebenen Funktionen aus sprachsoziologischer Sicht.

Der sprachliche Varietätenreichtum des Aostatals wird gemeinhin anhand der drei dominierenden Idiome Italienisch, Frankoprovenzalisch (patois) und Französisch illustriert. Die Mehrsprachigkeit unserer Grenzregion im äußersten Nordwesten Italiens stützt sich jedoch bekanntlich auf zwei weitere Idiome, wobei in diesem Beitrag vom Piemontesischen die Rede sein soll, dessen Präsenz bzw. Wirkung v.a. im südöstlichen Bereich der das gesamte Aostatal entwässernden Doire Baltée/Dora Baltea, in der sogenannten Basse Vallée spürbar wird. ${ }^{2}$

In innersprachlichem Zusammenhang ist die Rolle des Piemontesischen im Aostatal vereinzelt kontaktlinguistisch behandelt worden, so etwa bezüglich seines Einflusses auf die Morphosyntax (z.B. Verwendung von Artikel + Possessivum in der Basse Vallée bis Montjovet nach piemontesischem Vorbild vs. sporadischer Einsatz des Artikels in der Haute Vallée) ${ }^{3}$ oder das Lexikon ${ }^{4}$ der valdostanisch-frankoprovenzalischen Dialekte der Basse Vallée. Zur Bezeichnung von frz. baratte "Butterfaß"5 etwa ist in der Haute Vallée ist der Typ borée 6 üblich, in der Basse Vallée und im Piemont wird hingegen der Typ beus ${ }^{7}$ verwendet. Marco Perron synthetisiert die piemontesischen

1 Der vorliegende Beitrag stellt eine überarbeitete und erweiterte Übersetzung eines beim Rëscontr Antërnassional dë Studi an sla Lenga e la Literatura Piemontèisa ch'a fâ sëddes (Quinsnè/Quincinetto, 8.-9. Mai 1999) in italienischer Sprache gehaltenen Vortrags dar (vgl. dazu Bauer im Druck). Eine Kurzfassung in piemontesischer Sprache findet sich in Bauer 1998c. Unser Dank gilt an dieser Stelle dem hauptverantwortlichen Herausgeber der Tagungsakten von Quincinetto, Gianrenzo P. Clivio (Universität Toronto), der uns freundlicherweise die Rechte zur vorliegenden Publikation überlassen hat!

Siehe dazu Abb. 1, PP. 17-24. Zum hier nicht näher behandelten Walserdeutschen dreier Gemeinden des Lystales (Abb. 1, PP. 52-54) vgl. Zürrer 1999.

3 Vgl. Keller 1958, 141-142.

4 Vgl. Favre 1995, 22.

5 Zur Etymologie: griech. PRATTEIN "handeln" > 16. Jh. mfr. barattrer "Butter machen" > mfr., nfr. baratte "Butterfaß" (FEW IX, 330-333). W. v. Wartburg fügt hinzu, daß Wörter, bei denen eine Kampfhandlung im Vordergrund steht, wohl aus anord. BARATTA ("Kampf", REW 943) entlehnt sind (vgl. auch FEW XXII/1, 304-306). Die unsicheren Angaben des EWFS $(1928,78)$ bringen arab. BARRÂDA [?] "Gefäß" ins Spiel.

6 < griech. BŪTYRUM/BŪTŪRUM "Butter" (FEW I, 663-665, REW 1429).

7 <*BUTTIA "Faß”, REW 1425, vgl. vogesisch (Uriménil) beusse "baratte" (FEW I, 658-660). 
Einflüsse auf den patois wie folgt: "[...] les formes piémontaises pénètrent de plus en plus et le patois est souvent fort mélangé de traits piémontais." 8

In außer- oder soziolinguistischer Hinsicht jedoch gibt es bislang kaum systematische Betrachtungen zum Status und zu allfälligen Funktionen des Piemontesischen in seiner nördlichen Grenzregion. Im vorliegenden Beitrag soll versucht werden, folgende Fragen, die uns in diesem Zusammenhang von Interesse scheinen, zu beleuchten: Welche Rolle spielte das Piemontesische im Rahmen der historischen Entwicklung der valdostanischen Mehrsprachigkeit? Seit wann sind piemontesische Einflüsse belegt? Von wem/mit wem und in welchen Anwendungsbereichen wird heute noch Piemontesisch gesprochen? ${ }^{9}$ Wie sehen etwaige Zukunftsperspektiven des Piemontesischen im Aostatal aus?

Schon 1845 hatte der Stadtsekretär von Aosta, Laurent Pléoz explizit darauf verwiesen, daß die Sprechergemeinschaften jener Siedlungen des Haupttales, die zwischen Châtillon ${ }^{10}$ und der piemontesischen Grenze vor Ivrea liegen, u.a. auf Grund ihrer Handelskontakte mit den südlichen Nachbarn des Piemontesischen gemeinhin mächtig wären. ${ }^{11}$ Attilio Zuccagni-Orlandini wiederum ging 1864, also wenige Jahre nach vollzogener Einigung Italiens, auf die Stellung des Piemontesischen innerhalb der valdostanischen Sprachlandschaft ein, wobei seine Ausführungen, was zunächst überraschen mag, nicht nur den südöstlichen Talbereich ${ }^{12}$, sondern auch die Hauptstadt Aosta und die westlich davon gelegene Haute Vallée ${ }^{13}$ betrafen:

[...] a Cormayeur poi si parla una lingua, che partecipa del francese, dell'italiano e del piemontese, originata manifestamente dalla promiscuanza dei forestieri che vi dimorano nei mesi estivi, e provenienti dalle precitate contrade. [...] Nel Mandamento di Verres odesi il consueto amalgama di latino borgognone e piemontese [...] Fermiamoci finalmente nel centro del Circondario, ove appunto siede Aosta suo capoluogo, e cosi in quella città come nei circonvicini paesi il popolo risponderà alle nostre domande o con gergo impastato di voci galliche latine e piemontesi, o con impuro francese. ${ }^{14}$

Der sprachliche Einfluß (nord-)westlich der Basse Vallée erklärt sich v.a. aus der seit dem 18. Jahrhundert stetig steigenden Immigration von Piemontesen in die Hauptstadt Aosta sowie in die kleineren Handelszentren im Haupttal. Schon um 1830 stammte rund ein Fünftel der stadt-aostanischen Familien aus dem Piemont, während das lokale Periodikum Le Valdôtain im Jahr 1892 ein ganz und gar von den südlichen Nachbarn dominiertes Zukunftsszenario der Region zeichnete: "Se l'immigrazione

8 Perron 1995, 14; für weitere innerlinguistische Kontaktphänomene vgl. Favre im Druck und Grassi 1995.

9 In Anlehnung an die "berühmte" Fishman-Formel "Who speaks what Language to whom and when?" (1965).

10 Der Ortsdialekt von Châtillon (Abb. 1, P. 18) selbst ist übrigens nach Keller $(1958,142)$ eher vom StadtAostanischen (Abb. 1, P. 10) als vom Piemontesischen geprägt.

11 Vgl. den Abdruck des entsprechenden Zitats in Bauer 1997, 5; Vorlage: RAI 1993, 19.

12 Im Zitat wird die Ortschaft Verrès genannt, siehe Abb. 1, P. 23.

13 Im Zitat mit Courmayeur angesprochen, siehe Abb. 1, P. 51.

14 Zuccagni-Orlandini $1864,30-31$. 
piemontese, che ha già invaso tutte le borgate, avanza verso le montagne $[. .$.$] tra cin-$ quant'anni non rimarrà più nulla della Valle d'Aosta di un tempo." 15 Rund 40 Jahre später skizzierte Werner Walser (ebenfalls mit Blick auf die Immigrationsströme aus dem Piemont) das sprachliche Verhältnis der Valdostaner zum Piemontesischen folgendermaßen, wobei hier besonders auf den im Schlußsatz des Zitats angesprochenen, offensichtlich so empfundenen Prestige-Unterschied zwischen Piemontesisch und Frankoprovenzalisch hingewiesen sei:

Leichter jedoch als den Gebrauch der italienischen Schriftsprache erwirbt sich der Valdostaner die Kenntnis des Piemontesischen. Mit piemontesischen Einwanderern unterhält er sich meist ohne Schwierigkeit. Je häufiger die Einwanderung aus den Provinzen piemontesischer Zunge und je enger der Kontakt mit diesen benachbarten Gegenden wird, desto größer wird auch die Gefahr, die dem 'patois' von dieser Seite droht. Man gewinnt auch, besonders bei jungen Leuten, mehr und mehr den Eindruck, daß sie das Piemontesische dem 'patois' gegenüber als überwertig empfinden. ${ }^{16}$

Heute sind übrigens rund $40 \%$ der valdostanischen Bevölkerung außerhalb der Region geboren, ein Drittel davon stammt aus dem Piemont. Spitzenwerte piemontesischer Einwanderung sind, abgesehen von Aosta Stadt, erwartungsgemäß in der Basse Vallée zu finden, wobei der Grenzort Pont-Saint-Martin ${ }^{17}$, der von der Hauptstadt Aosta bereits $52 \mathrm{~km}$, von der nordwest-piemontesischen Industriestadt Ivrea jedoch lediglich $17 \mathrm{~km}$ entfernt liegt, mit 56,3\% Zuwanderern (Stand Volkszählung 1981) den absoluten Maximalwert verbucht. ${ }^{18}$

Gegen Ende des 19. Jahrhunderts, also zu einer Zeit, als die Staatssprache Italienisch (als Dach- bzw. Schriftsprache) das Französische aus den wichtigsten öffentlichen Bereichen wie Schulen, Gericht, Verwaltung und Presse bereits verdrängt hatte, wurde nun auch das Piemontesische als Gefahr für die historisch gewachsenen Regionalidiome Französisch und Frankoprovenzalisch, aber auch für das Italienische selbst angesehen. Tullio Omezzoli erwähnt in diesem Zusammenhang

[...] la tendenza del piemontese a sostituirsi, nelle relazioni interpersonali, al francese (oltre che al patois) e a rivendicare lo spazio che $i$ valdostani avrebbero volentieri concesso all'italiano. [...] E curioso come, nei decenni in cui è stato più vivo l'allarme-piemontese, nessuno in valle d'Aosta abbia espresso il timore che questo dialetto favorisse surrettiziamente l'italiano, o abbia pensato che esso fosse integrato in un sistema piemontese-italiano, al modo in cui si andava profilando [...] un sistema patois-francese. Il piemontese si configurava come ostile contemporaneamente all' $i t a l i a n o$ e al francese. ${ }^{19}$

\footnotetext{
15 Vgl. Omezzoli 1995b, 142-143, 176.

16 Walser 1937, 4.

17 Siehe Abb. 1, P. 24.

18 Für weitere demographische Details vgl. Bauer 1999a, 238-268.

19 Omezzoli 1995c, 50-52.
} 
Die von Kreisen valdostanischer Klerikal-Intellektueller um die Jahrhundertwende manifestierte Angst vor einer Vereinnahmung des patois und des als damit verbündet empfundenen Französischen durch das Piemontesische kommt auch in den folgenden Belegen zum Ausdruck. Abbé Frutaz rief im Jahr 1897 als Redakteur des Kirchenblattes Duché d'Aoste seine Landsleute dazu auf, die Pflege des Französischen nicht zu vernachlässigen und sich ihres patois nicht zu schämen, und warnte mit folgenden Worten: "Tra cinquant'anni [il popolo valdostano] sarà seppellito, e sulla sua lapide campeggerà un'epigrafe in piemontese!" 20 Abbé Cerlogne, welcher sich u.a. durch die Erstellung einer Grammatik und eines Wörterbuchs um den Ausbau des Frankoprovenzalischen verdient gemacht hatte, hegte die Befürchtung, daß der dialecte (gemeint ist der frankoprovenzalische patois) völlig vom Piemontesischen vereinnahmt werden könnte und daß dadurch auch das Französische an Terrain verlieren könnte:

Le dialecte plus coulant, dominant dans la Vallée, aura alors tout envahi. Sauf que, par manque de patriotismè, ne soit envahi lui-même par le piémontais, qui tend à se populariser dans notre vallée. Et alors nous perdrions, ensemble avec le 〈dia-lecte〉, CE que tout vrai valdôtain a toujours eu de plus cher: la langue française. ${ }^{21}$

Die Tatsache schließlich, daß die Sprachgewohnheiten der Valdostaner zu Beginn des 20. Jahrhunderts zwar domänenspezifisch differenziert, die aktiven wie passiven Sprachkompetenzen jedoch bisweilen zumindest vierstufig ausgeprägt waren und neben Französisch, Italienisch und Frankoprovenzalisch je nach Einsatzbereich auch das Piemontesische umfassten, kann durch ein Zitat von Abbé Petigat belegt werden, der die sprachlichen Usancen seiner Ministranten im Jahr 1911 wie folgt beschreibt: "Les gamins qui me servent la messe se disputent entre eux en piémontais, me répondent en français, puis à l'école réciteront en italien et, en famille, causeront patois." 22 In den walserdeutschen Gemeinden des ebenfalls im Einzugsgebiet der piemontesischen Verkehrssprache gelegenen Lystales ${ }^{23}$ ist für diese Zeit von einer Vier- bis Fünfsprachigkeit Deutsch-Piemontesisch-Französisch-Italienisch und mit Einschränkungen Frankoprovenzalisch auszugehen: "A Issime, gli abitanti, fra di loro parlano già un gergo intedescato; ma tutti parlano il dialetto piemontese, il francese-valdostano, e non troppo attempati bastanamente l'italiano."24

Die heutige Situation des Piemontesischen im Aostatal ist mit der soeben dokumentierten "Hochkonjunktur" der Jahrhundertwende freilich nicht mehr vergleichbar. In Nachwirkung der faschistischen Sprachpolitik, die v.a. auf die Eliminierung des

\footnotetext{
${ }^{20}$ Zitiert nach Omezzoli 1995a, 59-60; [Anmerkung RB].

21 Cerlogne 1907, zitiert nach dem Neudruck 1995, 6.

22 Auszug aus Le Duché d'Aoste vom 26.4.1911, zitiert nach RAI 1993, 34.

23 Siehe wiederum Abb. 1, PP. 52-54.

24 Aus einem Reiseführer von 1904, zitiert nach Zürrer 1999, 98; zum individuellen Mehrsprachigkeitsgrad valdostanischer Sprecher siehe die Farbgraphik in Bauer 1999a, 442.
} 
Französischen und auf die Zurückdrängung des Frankoprovenzalischen ausgerichtet war, mußte auch das Piemontesische Federn lassen. Im Vergleich zum staatstragenden Idealbild eines vom Regime als Modell für alle Sprecher suggerierten StandardItalienisch, wurde das Piemontesische (wie alle übrigen auf italienischem Territorium vertretenen regionalen Varietäten) als "bloßer Dialekt" geringgeschätzt, verlor somit an Prestige und mithin an Frequenz. Die in metalinguistischer Hinsicht (bis heute v.a. von regionalistischen Kreisen) empfundene Nähe des Piemontesischen zum Französischen impliziert(e) einen entsprechenden Abstand zum Italienischen ${ }^{25}$ und stand daher auch aus dieser Sicht im Widerspruch zum "Zeitgeist". Gute zehn Jahre nach Ende des 2. Weltkriegs sind in der Tat nur mehr wenige Einsatzbereiche übrig, in denen sich Valdostaner des Piemontesischen bedienen. Eine dieser potentiellen Gesprächssituationen wird im folgenden Zitat exemplarisch skizziert:

En effet, si aujourd'hui le paysan valdôtain veut vendre une vache dans un des nombreux marchés de bétail de la vallée, il lui faut parler piémontais, parce que le marchand de bétail piémontais ne lui parle que dans ce dialecte; si le paysan valdôtain ne le parle pas, il ne vendra guère sa bête. Voilà pourquoi les parlers valdôtains actuels fourmillent de mots piémontais, qui, souvent aussi, sont radoubés à la valdôtaine. [...] le bourg de Pont-Saint-Martin ne parle plus que piémontais, et dans les parlers de la Vallaise ainsi qu'à Donnas, à Bard et à Verrès, le dialecte valdôtain est en pleine décomposition. Cette évolution est fort regrettable, mais ne peut être arrêtée. ${ }^{26}$

Hans-Erich $\operatorname{Keller}^{27}$ bezieht sich also ausschließlich auf die Basse Vallée, wo das Piemontesische auch heute noch eine gewisse Vitalität verbuchen kann, auch wenn dies meist zu Lasten des frankoprovenzalischen patois geht. Im Jahr 1967 wurden 7.500 valdostanische Volksschüler einer gezielten Befragung unterzogen, aus der hervorging, daß noch in knapp 4\% aller Familien Piemontesisch gesprochen wurde. ${ }^{28}$ Auch in diesem Zusammenhang sei nochmals explizit unterstrichen, daß die betroffenen Sprecher hauptsächlich im Ort bzw. in den Gemeinden nordwestlich der Ortschaft Pont-Saint-Martin siedeln, die schon zu Kaiser Konstantins Zeiten im 4. Jahrhundert als Grenzort zwischen den gallischen Provinzen Alpes Graiae et Poeninae und den zur Italia gehörenden Alpes Cottiae fungierte.

Im Rahmen unserer eigenen Ende der 80er-Jahre durchgeführten Feldforschungen schilderten die Informanten aus Pont-Saint-Martin die sprachliche Lage ihres Heimatortes wie folgt:

\footnotetext{
${ }^{25} \mathrm{Vgl}$. Bauer 1999b, 78.

26 Keller 1959, 138. Zur Lokalisierung zweier im Zitat erwähnter Ortschaften siehe Abb. 1, P. 23 (Verrès), P. 24 (Pont-Saint-Martin).

$27 \dagger 1999$, vgl. Baldinger 2000 .

${ }^{28}$ Vgl. Bétemps 1972, 9 und Martin 1982, 58.
} 
1. Nella Bassa Valle, specialmente a Pont, si parla poco patois e pochissimo francese, predominano l'italiano e il piemontese.

2. Le français des locaux est influencé grandement par le piémontais et par l'italien.

3. On est aux confins de la région, où il n'y a pas un vrai dialecte, mais un ensemble des dialectes de la Basse Vallée et du piémontais, même si la plupart de la population entre elle parle italien. ${ }^{29}$

Unseren über 100 sprachsoziologischen Einzelenqueten können noch weitere Daten entnommen werden, die über die heutige Situation des Piemontesischen im Aostatal Auskunft geben. Ein Drittel aller von uns berücksichtigten 24 Meßpunkte kommt in der Basse Vallée zu liegen. ${ }^{30}$ Die Informantenangaben wurden hauptsächlich nach den in der Soziolinguistik etablierten Variablen Geschlecht, Alter, Schulbildung und Beruf statistisch ausgewertet und graphisch aufbereitet, wobei sichergestellt war, daß die Abweichungen unserer Stichprobe von der effektiven demographischen Realität innerhalb einer Toleranz von $2 \%$ verblieben.

Was die aktive Sprachkompetenz der hier berücksichtigten 32 Gewährsleute betrifft, so fällt zunächst der Umstand ins Auge, daß Männer ihre Piemontesischkenntnisse generell höher einstufen als Frauen (jeweils laut Selbsteinschätzung der Informanten): $20 \%$ der Frauen und immerhin 35\% der Männer meinten, mittelmäßig, gut oder sehr gut Piemontesisch sprechen zu können, während insgesamt gesehen rund $70 \%$ aller Informanten kaum bis gar keine entsprechenden aktiven Kenntnisse zu Protokoll gaben. Bezüglich der passiven Kompetenzen, die sich in erster Linie auf das Hörverstehen (und in Ermangelung leicht verfügbarer Lektürecorpora nur periphär auf das Lesen) bezogen, fielen die erhobenen Werte erwartungsgemäß um vieles höher aus, so daß man pauschalierend davon ausgehen kann, daß die valdostanische Bevölkerung der Basse Vallée einem piemontesischen Diskurs in der Regel ohne größere Verständnisprobleme folgen kann. Ein Vergleich dieser Daten mit den für das gesamte Aostatal erzielten Mittelwerten fördert zwei interessante Erkenntnisse zu Tage: Erstens übersteigen die Piemontesisch-Kompetenzen der Sprecher aus der Basse Vallée jene aller Valdostaner um ca. 25\% und zweitens nehmen die Frankoprovenzalisch-Kenntnisse mit steigender Piemontesisch-Kompetenz dermaßen $a b$, daß im süd-östlichen Talbereich nicht mehr der über die gesamte Region gesehen vorherrschende patois, sondern das Italienische als "dominante Sprache" in Erscheinung tritt.

In welchen Anwendungsbereichen werden nun die Piemontesisch-Kompetenzen umgesetzt? Ein eigener Bereich unseres Fragebuchs war der Domäne "Haus/Familie" gewidmet. Der Auswertung der erhobenen Antworten können auch diachronisch relevante Informationen entnommen werden, da die Gewährsleute einerseits nach der Familiensprache ihrer Kindheit und andererseits nach der heute zu Hause verwendeten Sprache befragt wurden. Während rund $10 \%$ der von uns interviewten 106 Sprecher

\footnotetext{
29 Bauer 1999a, 266-267.

${ }^{30}$ Siehe Abb. 1, PP. 17-24.
} 
angaben, zur Zeit ihrer Kindheit zu Hause noch Piemontesisch gesprochen zu haben (sei es nun ausschließlich oder zumindest abwechselnd mit Italienisch und Frankoprovenzalisch), fehlte das Piemontesische in den Antworten zur 1986/87 üblichen Familiensprache fast zur Gänze. Lediglich zwei Informanten nannten Italienisch und Piemontesisch als zu Hause verwendete Sprachen, wobei in beiden Fällen der jeweilige Ehepartner aus dem Piemont stammte. Für beide Zeiträume erwies sich übrigens der patois mit durchschnittlichen Gebrauchswerten von rund 65-70\% als das bei weitem dominierende Idiom. Eine Kreuzung der Antwortwerte zum "gestrigen" wie zum "heutigen" Sprachgebrauch in den Familien erlaubt uns nun, die im Laufe von ein bis zwei Generationen verbuchten Frequenzverluste bzw. -gewinne herauszuarbeiten. In Abbildung 2 sind die Ergebnisse in Form eines Balkendiagramms dargestellt: Man sieht zunächst, daß in den valdostanischen Familien heute praktisch nur mehr Frankoprovenzalisch und/oder Italienisch gesprochen wird. ${ }^{31}$ Die Piemontesisch-Sprecher von "gestern" sind zum überwiegenden Teil (57\%) zum Italienischen, zu 14\% zum patois abgewandert. ${ }^{32}$ Ein Vergleich der Cluster Frankoprovenzalisch und Italienisch zeigt, daß der patois im Generationensprung 31\% ehemalige Italienischsprecher an sich ziehen konnte, während in umgekehrter Richtung nur $17 \%$ einstige patoisants zum Italienischen abwanderten. Sogesehen sind Frankoprovenzalisch-Sprecher ihrem Familienidiom gegenüber bei weitem treuer als Italophone.

Unsere Frage nach der Muttersprache der Eltern der Informanten wurde zu 7\% mit der Antwort Piemontesisch quittiert. In weiteren 15\% der Antworten wurde das Piemontesische gemeinsam mit den drei in Frage kommenden Konkurrenzidiomen genannt. Hatten die Elternteile unserer Gewährsleute Italienisch und Frankoprovenzalisch zur Muttersprache, so setzte sich als Familiensprache schlußendlich immer das Italienische durch, war jedoch der patois in Kombination mit Französisch oder Piemontesisch als Sprache der Mutter oder des Vaters involviert, so behielt dieser in der Regel die Überhand. Aus diesen Zusammenhängen läßt sich, stark überzeichnend, eine Art Kräfteverhältnis zwischen den valdostanischen Idiomen ableiten, das gewisse Perspektiven für die Zukunft der valdostanischen Mehrsprachigkeit eröffnet: Italienisch erdrückt in jeder genannten Kombination das jeweilige Konkurrenzidiom. Der patois kann sich nur dann durchsetzen, wenn das Italienische nicht mit im Spiel ist. Piemontesisch und Französisch unterliegen in allen Mischantworten zugunsten ihrer Konkurrenten.

Die Eltern unter unseren Informanten (ca. 50\% aller Befragten) gaben uns Auskunft über zwei weitere Detailbereiche der Domäne Familie. In getrennten Fragen wurde erhoben, welche Sprache(n) sie mit ihren Eltern bzw. mit ihren Kindern verwen-

${ }^{31}$ Gebrauchswerte für weitere Idiome, die jeweils unter $10 \%$ liegen, sind übrigens in unserer Auswertung aufgrund zu niedriger Zellenhäufigkeit unberücksichtigt geblieben.

32 Siehe Abb. 2, Verteilung der gelben Balken. Die restlichen gut 30\% beziehen sich auf Mischantworten, die aus Gründen statistischer Unterrepräsentiertheit hier nicht vermerkt sind. 
deten. Die Kreuzung der beiden Antwortserien sollte nun, im Sinne der schon auf Abbildung 2 erprobten diachronen Perspektive, weitere Erkenntnisse zur Frequenzentwicklung des Piemontesischen im Generationensprung zu Tage fördern. Es ergibt sich das auf Abbildung 3 dargestellte Diagramm, aus dem lediglich Relationen und nicht etwa Quantitäten hervorgehen. So bezieht sich der hohe gelbe Balken im Italienisch-Cluster auf $100 \%$ derer, die angaben, mit ihren eigenen Eltern Piemontesisch zu sprechen/gesprochen zu haben, sagt aber nichts über ihre Anzahl aus. Wir sehen jedoch, daß mittlerweile alle betroffenen Sprecher mit ihren eigenen Kindern nicht mehr Piemontesisch, sondern ausschließlich Italienisch sprechen. Der (Total-)Rückzug des Piemontesischen aus dem Bereich der valdostanischen Familien kommt also auch in dieser Analyse deutlich zum Ausdruck.

Zur Stützung der bisher eruierten Tendenzen soll abschließend noch auf die Sprachverwendung beim Einkauf eingegangen werden. 39 Informanten gaben an, ihre Einkäufe regelmäßig auch in der piemontesischen Industriestadt Ivrea zu tätigen. Als dort einzusetzende Idiome kommen nur mehr Italienisch und Piemontesisch in Betracht. Die nach den Variablen Geschlecht und Alter errechneten Antwortverteilungen sind in Abbildung 4 tabellarisch zusammengestellt. 10\% der von uns in diesem Zusammenhang interviewten Frauen und 20\% der Männer nannten das Piemontesische als zumindest fallweise (i.e. in Kombination mit Italienisch) eingesetzte Einkaufssprache, weitere $10 \%$ der Gewährsmänner versicherten, beim Einkauf in Ivrea immer Piemontesisch zu sprechen. Ein Blick auf die Altersstruktur dieser Sprecher untermauert die These, daß es sich auch bei dieser domänenbezogenen Nische um evidentes Rückzugsgebiet handelt, waren doch alle betroffenen Piemontesisch-Sprecher zum Zeitpunkt der Umfrage bereits älter als 45 Jahre (i.e. vor 1940 geboren). Die jüngeren Sprechergenerationen gaben unisono an, ihre Einkäufe in Ivrea ausschließlich auf Italienisch abzuwickeln. Sogesehen paßt auch die Aussage eines unserer Informanten ins Bild, der auf die Frage, inwiefern sich der frankoprovenzalische patois der älteren von jenem der jüngeren Generation unterscheide, antwortete: "Nel dialetto dei giovani si sentono molte parole italiane, i vecchi usano più parole piemontesi."

Wurde das Piemontesische vor 100 Jahren noch als Bedrohung für die übrigen im Aostatal verwendeten Idiome angesehen, so läuft es heute selbst Gefahr, aus der valdostanischen Sprachlandschaft zu verschwinden. Deutlich spürbar bleibt sein Einfluß lediglich in Form lexikalischer, morphosyntaktischer und phonetischer Piemontesismen im Frankoprovenzalischen bzw. Regionalitalienischen v.a. älterer Sprecher.

\section{Bibliographie}

BALDINGER, Kurt (2000): Nécrologie: Hans-Erich Keller (1922-1999). In: Revue de Linguistique Romane 64, 311-314.

BAUER, Roland (1995): Plurilinguismus und Autonomie im Aostatal: Ergebnisse einer empirischen Untersuchung. In: Dieter Kattenbusch (Hg.), Minderheiten in der Romania, Wilhelmsfeld (Egert), (= pro lingua 22), 255-284. 
BAUER, Roland (1997): Die historische Entwicklung der Mehrsprachigkeit im Aostatal aus sprachsoziologischer Sicht: eine diachrone Rückschau samt Ausblick ins 21. Jahrhundert. In: Linguistica XXXVII, 3-25.

BAUER, Roland (1998a): Soziolinguistische Untersuchungen zur Mehrsprachigkeit im Aostatal. In: Iwar Werlen (Hg.), Mehrsprachigkeit im Alpenraum, Aarau/Frankfurt am Main/Salzburg (Sauerländer), (= Sprachlandschaft 22), 35-56.

BAUER, Roland (1998b): Aspetti del plurilinguismo in Valle d'Aosta / Vallée d'Aoste. In: Giovanni Ruffino (Hg.), Atti del XXI Congresso Internazionale di Linguistica e Filologia Romanza. Vol. V: Dialettologia, geolinguistica, sociolinguistica, Tübingen (Niemeyer), 31-45.

BAUER, Roland (1998c): Ël piemontèis an Valdosta da l'espansion a la regression (ins Piemontesische übersetzt von Camillo Brero). In: Piemontéis Ancheuj XVI1/184, 1-2.

BAUER, Roland (1999a): Sprachsoziologische Studien zur Mehrsprachigkeit im Aostatal: mit besonderer Berücksichtigung der externen Sprachgeschichte. Tübingen (Niemeyer), (= Beihefte zur Zeitschrift für romanische Philologie 296).

BAUER, Roland (1999b): Storia della copertura linguistica della Valle d'Aosta dal 1860 al 2000: un approccio sociolinguistico. In: Nouvelles du Centre d'Etudes Francoprovençales 39, 76-96.

BAUER, Roland (im Druck): Il piemontese in Valle d'Aosta dall'espansione alla regressione. In: Gianrenzo P. Clivio, Dario Pasero, Censin Pich (Hgg.), At XVI Rëscontr antërnassional dë Studi an sla Lenga e la Literatura Piemontèisa [Quinsnè, 8-9 magg 1999], Ivrea (La Slòira/Tipografia Ferraro).

BETEMPS, Alexis (1972): L'enseignement de la langue traditionnelle dans un pays en voie d'aliénation linguistique vers une rénovation des programmes et des méthodes: le laboratoire de langues. Aoste, (= Pubblicazioni del Gruppo di Ricerca in Sociolinguistica di Aosta 3).

CERlogne, Jean-Baptiste (1893): Premier essai. Petite grammaire du dialecte valdôtain avec traduction française, dédiée à Sa Majesté La Reine. Front Canavese (J.-B. Cerlogne).

Cerlogne, Jean-Baptiste (1907): Dictionnaire du patois valdôtain précédé de la petite grammaire du dialecte valdôtain. Aoste (Imprimerie catholique); Neudruck: Aoste (Le Château Edizioni) 1995.

EWFS: GAMILLSHEG, Emst (1928): Etymologisches Wörterbuch der französischen Sprache. Heidelberg (Winter).

FAVRE, Saverio (1995): Sur la zone médiane qui sépare et relie les parlers de la Haute et de la Basse Vallée d'Aoste. In: Nouvelles du Centre d'Etudes Francoprovençales 31, 18-32.

FAVRE, Saverio (im Druck): Le francoprovençal valdôtain et l'influence du piémontais. In: Gianrenzo P. Clivio, Dario Pasero, Censin Pich (Hgg.), At XV Rëscontr antërnassional dë Studi an sla Lenga e la Literatura Piemontèisa [Quinsnè, 9-10 magg 1998], Ivrea (La Slòira/Tipografia Ferraro).

FEW: VON WARTBURG, Walther (1922ff.): Französisches Etymologisches Wörterbuch. Eine Darstellung des galloromanischen Sprachschatzes. Band I: A-B. Tübingen (Mohr) 1948, [Bonn, 19221]; Band IX: Placabilis-Pyxis. Basel (Zbinden \& Co.) 1959; Band XXII/I: Matériaux d'origine inconnue ou incertaine. Bâle (Zbinden) 1976-1977.

FISHMAN, Joshua A. (1965): Who speaks what Language to whom and when? In: La Linguistique 1/2, 67-88.

Grassi, Corrado (1995): Tra Val d'Aosta e Piemonte: alcune note sui microsistemi dei dimostrativi. In: Nouvelles du Centre d'Etudes Francoprovençales 31, 57-63.

KELLER, Hans-Erich (1958): Etudes linguistiques sur les parlers valdôtains. Contribution à la connaissance des dialectes franco-provençaux modernes. Bern (Francke), (= Romanica Helvetica 66).

KELLER, Hans-Erich (1959): Structure des parlers valdôtains et leur position parmi les langues néo-latines. In: $L a$ Valle d'Aosta. Relazioni e comunicazioni presentate al XXXI Congresso Storico Alpino, Torino (Deputazione subalpina di storia patria), vol.I, 123-138.

MARTIN, Jean-Pierre (1982): Aperçu historique de la langue française en Vallée d'Aoste. Tradition et progrès. Aoste (Duc).

OMezzol, Tullio (1995a): Un giornale clericale. "Le Duché d'Aoste" (1894-1926). Aosta (Le Château Edizioni).

OMEzzoLI, Tullio (1995b): Lingue e identità valdostana. In: Stuart J. Woolf (Hg.), Storia d'Italia. Le regioni dall'Unità a oggi. La Valle d'Aosta, Torino (Einaudi), 137-202.

OMEzzoLI, Tullio (1995c): Alcune postille sulle lingue dei Valdostani. Aosta (Le Château Edizioni).

Perron, Marco (1995): Les isoglosses en Vallée d'Aoste. In: Nouvelles du Centre d'Etudes Francoprovença-les $31,13-17$.

RAI (1993) = Radiotelevisione Italiana, Siège Régional / Sede Regionale della Valle d'Aosta: Bilinguisme et plurilinguisme. Un entretien avec André Martinet. Aosta (Duc), (= Collana Cahiers / Quademi RAI 5). 
REW: MEYER-LÜBKE, Wilhelm $\left(1992^{6}, 1935^{3}\right)$ : Romanisches etymologisches Wörterbuch. Heidelberg (Winter) [1911-19201].

WALSER, Werner (1937): Zur Charakteristik der Mundart des Aosta-Tales. Aarau (Sauerländer).

ZUCCAGNI-ORLANDINI, Attilio (1864): Raccolta di dialetti italiani con illustrazioni etnologiche. Firenze (Tipografia Tofani).

ZÜRRER, Peter (1999): Sprachinseldialekte. Walserdeutsch im Aostatal (Italien). Aarau/Frankfurt am Main/Salzburg (Sauerländer), (= Sprachlandschaft 23 ).

\section{Povzetek}

\section{PIEMONTEŠKO NAREČJE V DOLINI AOSTE}

Sociolingvistično zasnovani prispevek obravnava včerajšnji in današnji položaj kakor tudi morebitne funkcije piemonteškega narečja v italijanski avtonomni deželi Aosti; v študijah večjezičnosti, ki jo ta dežela pozna, je bilo to narečje dozdaj najmanj upoštevano. Poskuša se odgovoriti na sledeča vprašanja: kakšno vlogo je imelo piemonteško narečje v zgodovinskem razvoju večjezičnosti v Aosti; od kdaj so ugotovljivi piemonteški jezikovni vplivi na govorjeni jezik v deželi; kdo, s kom, ob kakšni priliki, kdaj in zakaj se dandanes še govori piemonteško; kakšne so perspektive piemonteškega narečja.

Treba je upoštevati, da je bila raba piemonteškega narečja v Aosti še ob koncu 19. stoletja zelo močna in je prevladovala nad rabo francoščine in frankoprovansalščine; danes, torej nekako sto let kasneje, pa narečju grozi izginotje. Le v jugozahodnem delu doline, v t.i. Basse vallée, je mogoče ugotoviti znatne leksikalne, morfosintaktične in fonetične piemonteške prvine v frankoprovansalščini oziroma regionalni italijanščini zlasti starejše generacije. 


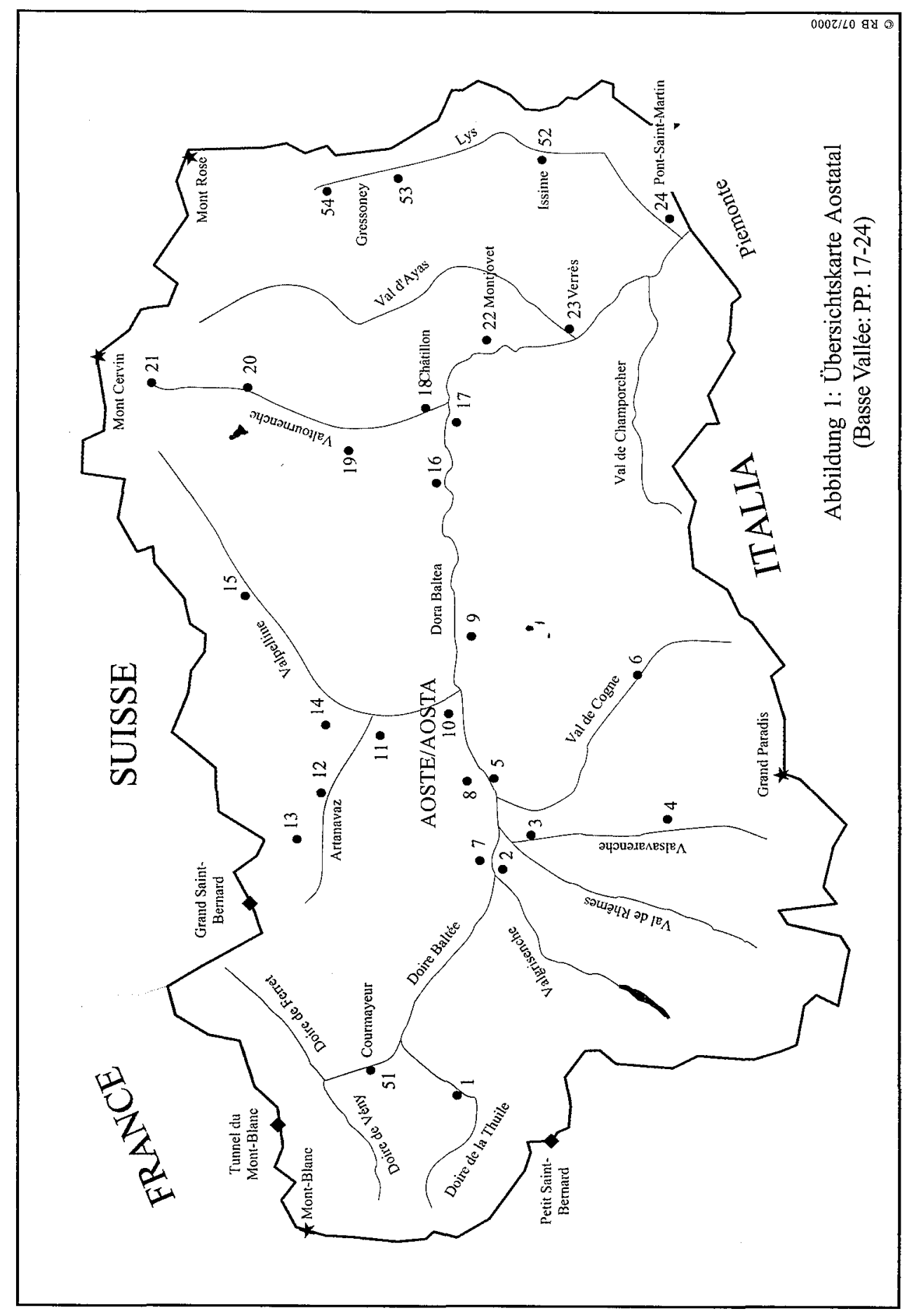




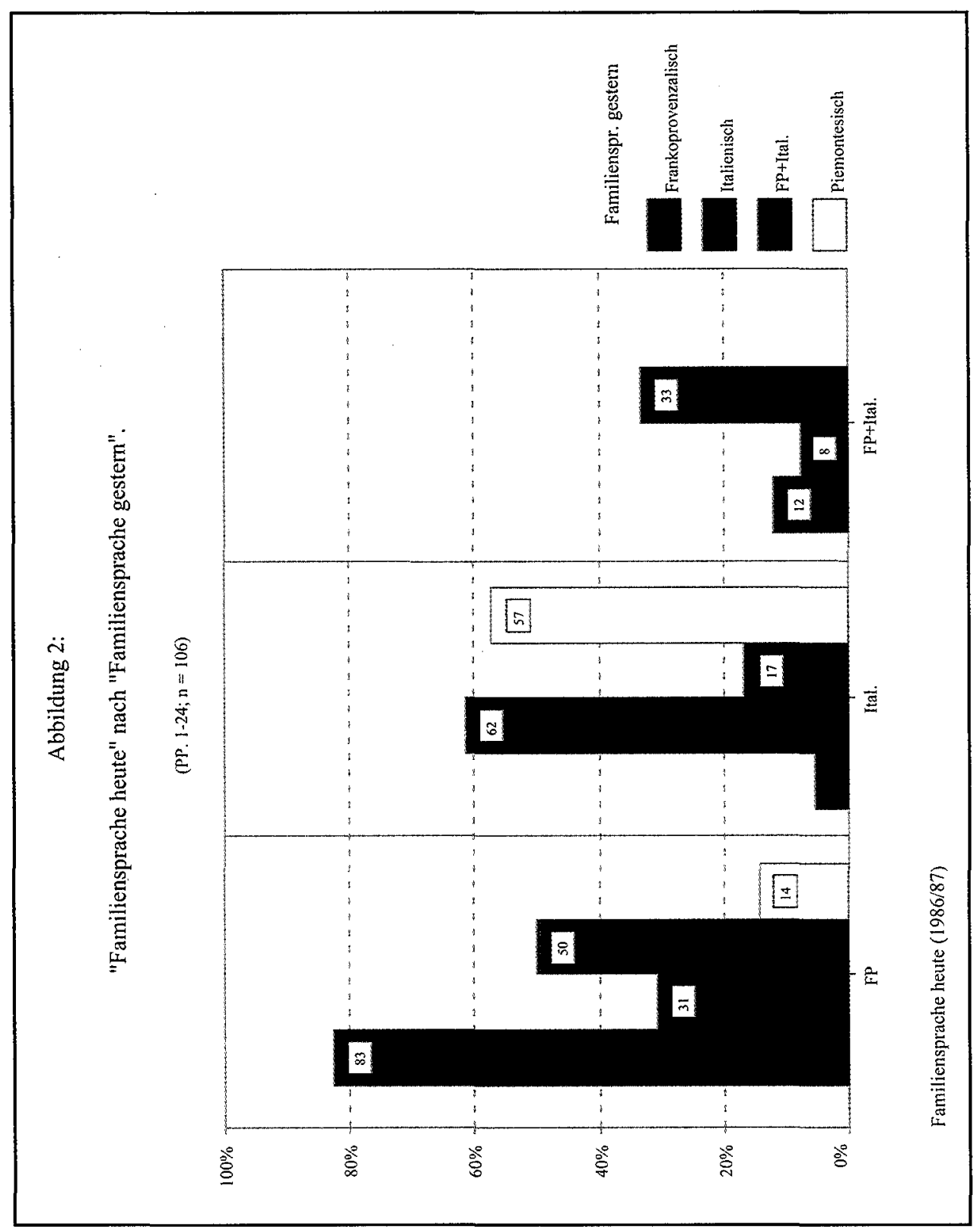




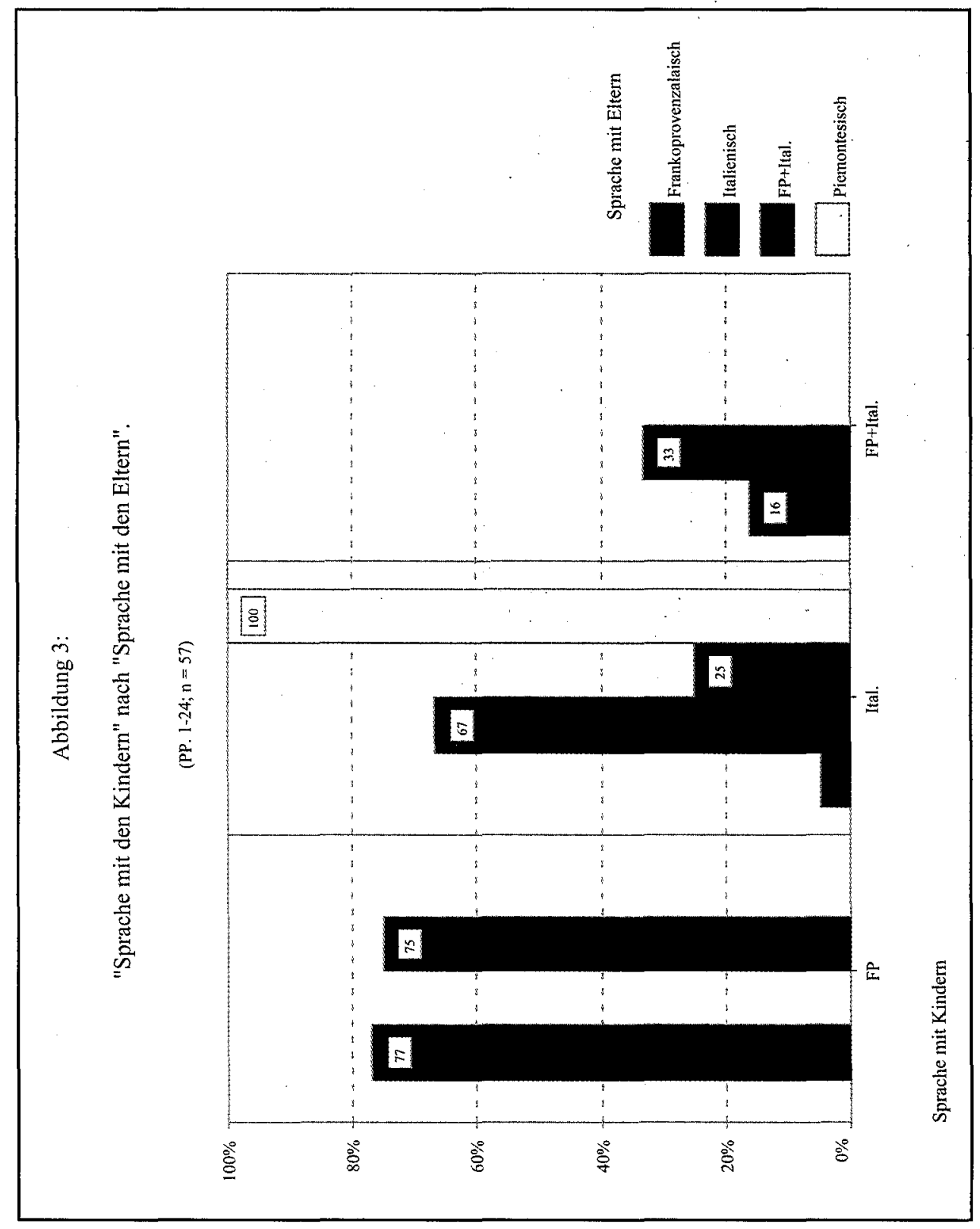




\begin{tabular}{|c|c|c|c|}
\hline \multirow{2}{*}{$\begin{array}{l}\text { Sprecher- } \\
\text { gruppen }\end{array}$} & \multicolumn{3}{|c|}{ E I N K A U F I N I V R E A } \\
\hline & Italienisch & Piemontesisch & Piem. +Ital. \\
\hline alle $(n=39)$ & $76,9 \%$ & $5,1 \%$ & $15,4 \%$ \\
\hline $\mathrm{m}$ & $65 \%$ & $10 \%$ & $20 \%$ \\
\hline$f$ & $89,5 \%$ & $\varnothing$ & $10,5 \%$ \\
\hline$<26$ Jahre alt & $100 \%$ & $\varnothing$ & $\varnothing$ \\
\hline $26-35$ Jahre alt & $100 \%$ & $\varnothing$ & $\varnothing$ \\
\hline $36-45$ Jahre alt & $100 \%$ & $\varnothing$ & $\varnothing$ \\
\hline $46-55$ Jahre alt & $\varnothing$ & $\varnothing$ & $100 \%$ \\
\hline $56-65$ Jahre alt & $50 \%$ & $\varnothing$ & $50 \%$ \\
\hline$>65$ Jahre alt & $50 \%$ & $50 \%$ & $\varnothing$ \\
\hline
\end{tabular}

Abbildung 4

Beim Einkauf in Ivrea verwendete Sprache(n).

(Mischantworten unter 5\% Gesamtaufkommen nicht berücksichtigt) 